F. Pustahija, N. Bašić \& S. Siljak-Yakovlev

\title{
Chromosome numbers and genome size data for some Balkan species
}

\begin{abstract}
Pustahija, F., Bašić, N. \& Siljak-Yakovlev, S.: Chromosome numbers and genome size data for some Balkan species. [In Kamari, G., Blanché, C. \& Siljak-Yakovlev, S. (eds), Mediterranean plant karyological data - 28]. - Fl. Medit. 28: 420-428. doi: 10.7320/FlMedit28.420

Chromosome numbers and metaphase plates are given for eight species, seven from Bosnia and Herzegovina (Alnus $\times$ pubescens, Erythronium dens-canis, Genista tinctoria, Leucanthemum vulgare, Melittis melissophyllum, Orchis mascula, Stachys recta), and one species from F.Y.R.O.M. (Scorzonera austriaca). Chromosome counts and genome sizes are discussed.

Keywords: karyology, Alnus, Erythronium, Genista, Leucanthemum, Melittis, Orchis, Stachys, Scorzonera, Bosnia and Herzegovina, F.Y.R.O.M.
\end{abstract}

1935. Alnus $\times$ pubescens Tausch. $-2 n=4 \mathrm{x}=28 \& 2 n=6 \mathrm{x}=42$ (Figs 1a \& 1b).

BH: Kreševo, schist, $43^{\circ} 49^{\prime} 33,35^{\prime}$ N, $18^{\circ} 02^{\prime}$ 28,19” E, 770 m a.s.1., 10 Nov 2007, private herbarium of N.B.

Alnus $\times$ pubescens Tausch. is a deciduous tree from Betulaceae family, considered as hybrid between black [Alnus glutinosa (L.) Gaertn] and grey alder [A. incana (L.) Moench]. According to relevant literature this taxon is registered in Belarus, Bosnia and Herzegovina, Croatia, the Czech Republic, Ireland, Latvia, Poland, Serbia and Sweden.

The tetraploid chromosome number $2 n=28$ is reported by Siljak-Yakovlev \& al. (2010), and octoploid $2 n=56$ by Helms \& Jørgensen (1925). The hexaploid chromosome number $2 n=42$ is given here for the first time. We confirm, also, a tetraploid $2 n=28$ chromosome number in the same population. Genome size of tetraploids is $2 \mathrm{C}=1.05 \mathrm{pg}$ (Siljak-Yakovlev \& al. 2010).

1936. Erythronium dens-canis L. $-2 n=2 \mathrm{x}=24$ (Fig. 1c).

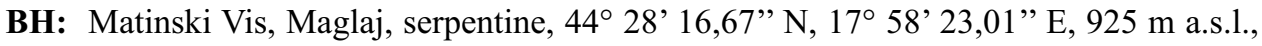
10 Apr 2008, private herbarium of F.P.

Erythronium dens-canis L. is a bulbous herbaceous perennial flowering plant (Liliaceae), native to central and southern Europe; the only naturally occurring species of genus in Europe. 
The diploid chromosome number $2 n=24$ confirms previous reports of Hrubý (1934), Delay (1947), Capineri \& al. (1978), Sopova \& Sekovski (1982), Rosselló \& al. (1987), Krichphalushi (1989), and García Martínez (2001), but differs from $2 n=4 \mathrm{x}=48$ (SiljakYakovlev \& al. 2010). The measured genome sizes are for tetraploids $2 \mathrm{C}=49.98 \mathrm{pg}$ (Siljak-Yakovlev \& al. 2010), then $2 \mathrm{C}=52.91 \mathrm{pg}($ Vesely $\&$ al. 2011) and $2 \mathrm{C}=59.80 \mathrm{pg}$ (Zonneveld \& al. 2005) for unknown ploidies.

1937. Genista tinctoria L. $-2 n=4 \mathrm{x}=96$ (Fig. 1d).

BH: Katranica, MuškeVode, Kladanj, serpentine, 44 ${ }^{\circ} 14^{\prime} 10,36^{\prime \prime}$ N, $18^{\circ} 34^{\prime}$ 46,62” E, 791 $\mathrm{m}$ a.s.1., 29 Sept 2016, private herbarium of F.P.

Genista tinctoria L. is a variable deciduous shrub of the family Fabaceae, native to Europe and western Asia, but widely cultivated as a horticultural species.

The tetraploid chromosome number $2 n=96$ established for one population growing on serpentine substrate is in accordance with previous data on this variable species (Semerenko \& Blazhevich 1979; Semerenko \& Shvets 1989; Siljak-Yakovlev \& al. 2010; Pustahija \& al. 2013), but diploids are more frequently determined (Čehov 1931; Tischler 1934; Santos 1945; Garajová 1959; Gadella \& Kliphuis 1966; Krusheva 1975; Semerenko \& Blazhevich 1979; Pogan \& al. 1982; Van Loon \& Setten 1982; Gallego Martín \& al. 1988; Cubas \& al. 1998). Siljak-Yakovlev \& al. (2010) and Pustahija \& al. (2013) estimated the genome size for tetraploids, $2 \mathrm{C}=3.34 \mathrm{pg}$.

1938. Leucanthemum vulgare (Vaill.) Lam. $-2 n=8 \mathrm{x}=72$ (Fig. 1e).

BH: Katranica, MuškeVode, Kladanj, serpentine, 44 14' 11,57’ N, $18^{\circ} 34^{\prime}$ 15,64” E, 832 $\mathrm{m}$ a.s.1., 14 Aug 2008, private herbarium of F.P.

Leucanthemum vulgare (Vaill.) Lam., Asteraceae, is a typical grassland perennial flowering plant, native to Europe and the temperate regions of Asia, introduced to North America, Australia and New Zealand.

Different chromosome numbers are given in the literature: $2 n=18$ (Žukova 1964; Polatschek 1966; Čuksanova \& al. 1968; Nagl \& Ehrendorfer 1974; Khandjian 1975; Morton 1977; Rostovtseva 1979; Teppner 1980; Strid \& Andersson 1985; Lippert \& Heubl 1989; Probatova \& al. 1989; Vogt 1991; Dempsey \& al. 1994; Albers \& Pröbsting 1998; Dmitrieva 2000; Probatova 2000; Vogt 2000; Gregor Hand 2009), $2 n=18+1 \mathrm{~B}$ (Khandjian 1975), $2 n=36$ (Žukova 1964; Čuksanova \& al. 1968; Morton 1977, 1981; Arohonka 1982; Marchi \& al. 1983; D’Ovidio 1984; Dmitrieva 1987; Parfenov \& Dmitrieva 1987; Parfenov \& Dmitrieva 1988b; Lippert \& Heubl 1989; Lavrenko \& al. 1991; Lavrenko \& al. 1992; Stepanov \& Muratova 1995; Lövkvist \& Hultgård 1999), $2 n$ $=36+1 \mathrm{~B}$ (Parfenov \& Dmitrieva 1988a; Dmitrieva 2000), $2 n=54$ (Parfitt 1981; Lippert \& Heubl 1989), $2 n=72$ (Lippert \& Heubl 1989; Siljak-Yakovlev \& al. 2010; Pustahija \& al. 2013), and $2 n=90$ (Favarger \& Villard 1965) for this aggregate, and our chromosome 
number determination of $2 n=72$ is in accordance with previous reports for octaploids. Pustahija \& al. (2013) estimated genome size for diploids $(2 \mathrm{C}=10.54 \mathrm{pg})$; Nagl \& Ehrendorfer (1974), Marchi \& al. (1983), Band (1983) and Pustahija \& al. (2013) for tetraploids $(2 \mathrm{C}=11.55,25.35,21.30$ and $20.20 \mathrm{pg}$, respectively); and Siljak-Yakovlev \& al. (2010) for octaploids $(2 \mathrm{C}=32.69 \mathrm{pg})$. Bai \& al. (2012) estimated $2 \mathrm{C}=10.20$ and 17.60 pg for unknown ploidy levels.

1939. Melittis melissophyllum L. $-2 n=2 \mathrm{x}=30$ (Fig. 1f).

BH: Katranica, Muške Vode, Kladanj, serpentine, $44^{\circ} 14^{\prime} 11,57^{\prime}$ N, $18^{\circ} 34^{\prime} 15,64$ ' E, $832 \mathrm{~m}$ a.s.1., 14 Aug 2008, private herbarium of F.P.

Melittis melissophyllum L., Lamiaceae, is herbaceous perennial flowering plant in the monospecific genus Melittis, native to central and southern Europe.

The diploid chromosome number $2 n=30$ is in accordance with the most available literature data (Bakšay 1958; Pogan 1982; Rosselló \& al. 1987; Tasenkevitch \& al. 1989; Siljak-Yakovlev \& al. 2010). However, the $2 n=24$ was also reported by Strid and Franzen (1981) and $2 n=30+1-2 B$ by Dobea $\&$ al. (1997). Genome size of diploids was $2 \mathrm{C}=0.95$ pg (Siljak-Yakovlev \& al. 2010).

1940. Orchis mascula (L.) L. $-2 n=2 \mathrm{x}=42$ (Fig. $2 \mathrm{~g}$ ).

BH: Mliništa, Glamoč, limestone, $44^{\circ} 14^{\prime}$ 08,37” N, $16^{\circ} 49^{\prime}$ 50,43” E, 1204 m a.s.1., 13 May 2008, private herbarium of F.P.

Orchis mascula (L.) L., Orchidaceae, is tuberous perennial herbaceous plant, native to Eurasia and North Africa.

The diploid chromosome number $2 n=42$ is stable for the species and coincides with data obtained by different authors (Hagerup 1938; Heusser 1938; Vermeulen 1949; Skalińska \& al. 1957; Gadella \& Kliphuis 1963; Kliphuis 1963; Averyanov \& al. 1985; D’Emerico \& al. 2002; Bernardos 2004). Genome size has not been estimated to date.

1941. Scorzonera austriaca Willd. $-2 n=2 \mathrm{x}=14$ (Fig. $1 \mathrm{~h}$ ).

F.Y.R.O.M.: Pčinja canyon, near Veles, May 2009.

Scorzonera austriaca Willd., highly polymorphic complex, is a perennial plant of Asteraceae family, native to the central and south-east Europe and Temperate Asia.

The diploid chromosome number $2 n=14$ counted here agrees with previously reported (Tarnavschi 1938; Sosnovec 1960; Dvorak \& Dadakova 1977; Brullo \& al. 1978; Strid \& Franzen 1983; Kuzmanov \& al. 1986; Stepanov 1992; Idei 1996). Estimated genome size for this species was $2 \mathrm{C}=10.68 \mathrm{pg}$ (Siljak-Yakovlev \& al. 2010). 


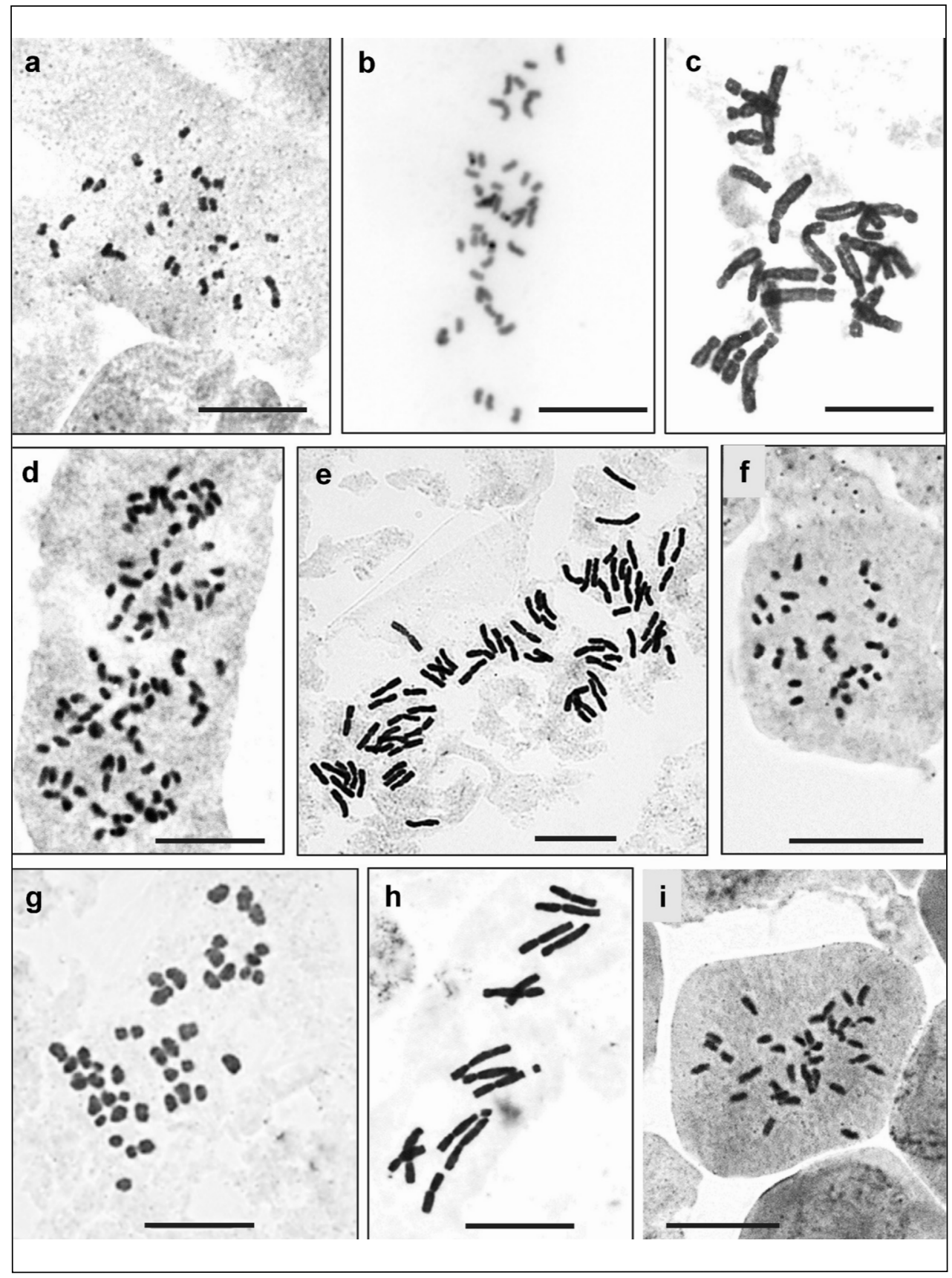

Fig. 1. Mitotic methaphase plates of: a, Alnus pubescens, $2 n=4 \mathrm{x}=28 ; \mathbf{b}$, Alnus pubescens, $2 n=6 \mathrm{x}$ $=42 ;$ c, Erythronium dens-canis, $2 n=2 \mathrm{x}=24 ; \mathbf{d}$, Genista tinctoria, $2 n=4 \mathrm{x}=96 ; \mathbf{e}$, Leucanthemum vulgare, $2 n=8 \mathrm{x}=72 ; \mathbf{f}$, Melittis melissophyllum, $2 n=2 \mathrm{x}=30 ; \mathbf{g}$, Orchis mascula, $2 n=2 \mathrm{x}=42 ; \mathbf{h}$, Scorzonera austriaca, $2 n=2 \mathrm{x}=14 ; \mathbf{i}$, Stachys recta, $2 n=2 \mathrm{x}=32+1 \mathrm{~B}$. - Scale bars $=10 \mu \mathrm{m}$. 
1942. Stachys recta L. $-2 n=2 \mathrm{x}=32+1 \mathrm{~B}$ (Fig. 1i).

BH: Katranica, Muške Vode, Kladanj, serpentine, $44^{\circ} 14 ' 10,12$ " N, $18^{\circ} 34^{\prime} 14,01$ " E, 824 m a.s.1., 14 Aug 2008, private herbarium of F.P.

Stachys recta L., Lamiaceae, is an herbaceous perennial plant, natively widespread from Europe to the Caucasus and Asia Minor.

The diploid chromosome number $2 n=32+1 \mathrm{~B}$ confirms previous reports for the variety chrysophea (Siljak-Yakovlev \& al. 2010; Pustahija \& al. 2013), but differs from $2 n=32$ (Pólya 1949), $2 n=32-34$ (Verlaque \& al. 1987), $2 n=34$ (Lang 1940; Favarger 1959; Koeva-Todorovska 1978; Kliphuis \& Wieffering 1979; Cusma Velari \& Lausi 1980; Pogan \& al. 1982; Van Loon \& Setten 1982; Baltisberger \& Lenherr 1984; Baltisberger 1988; Lippert \& Heubl 1989; Baltisberger 1990a, 1990b; Dobea \& al. 1997; Siljak-Yakovlev \& al. 2010), and $2 n=48$ (Delay 1947). Estimated genome size for species on limestone was $2 \mathrm{C}=1.73 \mathrm{pg}$ (Siljak-Yakovlev \& al. 2010), and $2 \mathrm{C}=1.83 \mathrm{pg}$ for variety chrysophea on serpentine (Siljak-Yakovlev \& al. 2010; Pustahija \& al. 2013).

\section{References}

Albers, F. \& Pröbsting, W. 1998: In Wisskirchen, R. \& Haeupler, H., Standardliste der Farn- und Blütenpflanzen Deutschlands. Bundesamt für Naturschutz \&Verlag Eugen Ulmer, Stuttgart.

Arohonka, T. 1982: Chromosome counts of vascular plants of the island Seili in Nauvo, southwestern Finland. - Ann. Univ. Turku., Ser. A, Biol.Geogr. 3: 1112.

Averyanov, L. V., Medvedeva, N. A. \& Serov, V. P. 1985: Chromosome numbers in the representatives of the family Orchidaceae from the Caucasus. - Bot. Zhurn. SSSR 70(7): 9991000. (In Russian).

Bai, C., Alverson, W. S., Follansbee, A. \& Waller, D. M. 2012: New reports of nuclear DNA content for 407 vascular plant taxa from the United States. - Ann Bot 110(8): 1623-1629.

Bakšay, L. 1958: The chromosome numbers of Ponto-Mediterannean plant species. - Ann. Hist.-Nat. Mus. Natl. Hungarici 50(Ser. nova 9): 121-125.

Baltisberger, M. \& Lenherr, A. 1984: Neue Chromosomenzahlen aus der Gruppe der Stachys recta L. und anderen, verwandten Artengruppen. - Ber. Geobot. Inst. ETH Stiftung Rübel 51: 39-62.

Baltisberger, M. 1988: Numeri cromosomici per la Flora Italiana: 1167-1184. - Inform. Bot. Ital. 20: 627-636.

— 1990a: Chromosomenzahlen einiger Pflanzen aus Jugoslawien. - Candollea 45: 439-446.

- 1990b: Zytologische Untersuchungen an einigen Arten aus Italien. - Arch. Bot. Ital. 66: 153-165.

Band, S. R. 1984: personal communication. - http://data.kew.org/cvalues/cvalOrigReference.html\#63.

Bernardos, S. 2004: Cytotaxonomic study of some taxa of the subtribe Orchidinae (Orchidoideae, Orchidaceae) from the Iberian Peninsula. - Israel J. P1. Sci. 52: 161-170.

Brullo, S., Majorana, G., Pavone, P. \& Terrasi, M. C. 1978: Numeri cromosomici per la Flora Italiana: 366-374. - Inform. Bot. Ital. 10: 46-55.

Capineri, R., D’Amato, G. \& Marchi, P. 1978: Numeri cromosomici per la Flora Italiana. 534-583. - Inform. Bot. Ital. 10: 421-465.

Čehov, V. 1931: Kariosistematičeskoe issledovanie tribusov Sophoreae Spreng., Podalariae Benth. i Genistae Bronn. sem. Leguminosae. - Izv. Tomsk. otd. Gos. russk. bot. obšć. 3(12): $121-131$. 
Cubas, P., Pardo, C., Sánchez-Mata, D. \& Cantó, P. 1998: Karyological and taxonomic notes on Genista L. (Papilionoideae, Leguminosae) from the Iberian Peninsula. - Bot. J. Linn. Soc. 128: $423-434$.

Čuksanova, N. A., Svešnikova, L. I. \& Aleksandrova, T. V. 1968: Materiali k kariologii semejstva Složnocvetnih. - Citologija 10(2): 198-206.

Cusma Velari, T. \& Lausi, D. 1980: Indagini cariologiche in Stachys recta L., S. labiosa Bertol., S. subcrenata Vis., S. fragilis Vis. - Stud. Geobot. 1(1): 149-155.

Delay, C. 1947: Recherches sur la structure des noyaux quiescent chez les Phanérogames. - Rev. Cytol. et Cytophysiol. Vég. 9(1-4): 169-222; 10(1-4): 103-229.

D'Emerico, S., Cozzolino, S., Pellegrino, G., Pignone, D. \& Scrugli, A. 2002: Heterochromatin distribution in selected taxa of the 42-chromosomes Orchis s.1. (Orchidaceae). - Caryologia 55: 55-62.

Dempsey, R. E., Gornall, R. J. \& Bailey, J. P. 1994: Contributions to a cytological catalogue of the British and Irish flora, 4. - Watsonia 20: 63-66.

Dmitrieva, S. A. 1987: Kariologicheskaja kharakteristika nekotorykh predstaviteley sem. slozhnocvetnykh (Asteraceae Dumort.) flory Belorussii. - Botanika (Minsk) 28: 23-33.

- 2000: Karyology of the flora of Byelarus. Pp. 42 in Thesis of the Diss. Doc. Biol. Sci. Minsk.

Dobea, C., Hahn, B. \& Morawetz, W. 1997: Chromosomenzahlen zur Gefässpflanzen-Flora Österreichs. - Linzer Biol. Beitr. 29(1): 5-43.

D’Ovidio, R. 1984: Numeri cromosomici per la Flora Italiana: 1011-1021. - Inform. Bot. Ital. 16: 251-260.

Dvorak, F. \& Dadakova, B. 1977: Reports. [In Löve, A. (ed.) IOPB chromosome number reports LVI]. - Taxon 26: 257-274.

Favarger, C. 1959: Notes de caryologie alpine. III. - Bul. Soc. Neuchâtel. Sci. Nat., Ser. 3, 82: 255-285.

— \& Villard, M. 1965: Nouvelles recherces cytotaxinomiques sur Chrysanthemum leucanthemum L. sens. lat. - Ber. Schweiz. Bot. Ges. (Bull. Soc. Bot. Suisse) 75: 54-79.

Gadella, T. W. J. \& Kliphuis, E. 1963: Chromosome numbers of flowering plants in the Netherlands. - Acta Bot. Neerlandica 12(2): 195-230.

— \& - 1966: Chromosome numbers of flowering plants in the Netherlands. II. - K. Akad. Wetenschap. Amsterdam Proc., Ser. C, 69(5): 541-556.

Gallego Martín, F., Anta, M. A. S. \& Andrés, F. N. 1988: Acerca de la cariología de algunas Genisteas del centro-occidente Español. - Lazaroa 9: 55-60.

Garajová, S. 1959: Prispevok k cytologickým pomerom flóry okolia Bratislavy. - (Diplom práca in litt.), Bratislava: 1-49.

García Martínez, X. R. 2001: Números cromosomáticos de plantas occidentales, 855-862. - Anales Jard. Bot. Madrid 58(2): 341.

Gregor, T. \& Hand, R. 2009: Chromosomenzahlen von Farn- und Samenpflanzen aus Deutschland 4. - Kochia 4: 37-46.

Hagerup, O. 1938: Studies on the significance of polyploidy. II. Orchis. - Hereditas 24(1-2): 258-263.

Helms, A. \& Jørgensen, C. A. 1925: Birkenepaa Maglemose. - Botanisk Tidskrift 39: 57-133.

Heusser, C. 1938: Chromosomenverhältnisse bei schweizerischen basitonen Orchideen. - Ber. Schweiz. Bot. ges. (Bull. Soc. Bot. suisse) 48: 562-599.

Hrubý, K. 1934: A contribution to the cytology and embryology of Erythronium dens-canis L. - Bull. Internatl. Acad. Tchèque Sci. Cl. Sci., Mat. et Méd. Prague 35: 124-132.

Idei, S., Kondo, K., Hong, D. \& Yangdei, Q. 1996: Karyotype of Scorzonera austri[a]ca Willd. of China by using fluorescence in situ hybridization. - Kromosomo 1996: 2893-2900.

Khandjian, N. S. 1975: New data on chromosome number in Tanacetum L. and Leucanthemum Mill. - Biol. Zurn. Armen. 28: 87-89. 
Kliphuis, E. 1963: Cytological observations in relation to the taxonomy of the orchids of the Netherlands. - Acta Bot. Neerlandica 12(2): 172-194.

— \& Wieffering, J. H. 1979: Reports. [In Löve, A. (ed.) IOPB chromosome number reports LXIV]. - Taxon 28: 398-400.

Koeva-Todorovska, J. 1978: Pontostachys - A new section of the genus Stachys L. - Phytologija (Sofia) 10: 33-40.

Krichphalushi, V. V. 1989: Sravnitelno-karyologicheskoe issledovanie prirodnykh populacij efemeroidov Karpat. Pp. 25-27 in Tesizy II Symp. Plant Karyology.

Krusheva, R. M. 1975: Reports. [In Löve, A. (ed.) IOPB chromosome number reports L]. - Taxon 24: 671-678.

Kuzmanov, B. A., Georgieva, S. B. \& Nikolova, V. A. 1986: Chromosome numbers of Bulgarian flowering plants. I. Fam. Asteraceae. - Fitologija 31: 71-74.

Lang, A. von, 1940: Untersuchungen über einige Verwandtschafts- und Abstammungsfragen in der Gattung Stachys L. auf cytogenetischer Grundlage. - Bibliotheca Bot. 29(18): 1-94.

Lavrenko, A. N., Serditov, N. P. \& Ulle, Z. G. 1992: Kariologicheskie osobennosti i rasprostranenie predstavitelej semejstva Asteraceae v Pechoro-Ilychskom Biosfernom Zapovednike. - Trudy Komi Nauchnogo Tsentra UrO AN SSSR 126: 52-64.

Lavrenko, A. N., Serditov, N. P. \& Ulle, Z. G. 1991: Chromosome numbers in some species of vascular plants from the Pechoro-Ilychsky Reservation (Komi ASSR). - Bot. Zhurn. (Moscow \& Leningrad) 76: 473-476.

Lippert, W. \& Heubl, G. R. 1989: Chromosomenzahlen von Pflanzen aus Bayern und angrenzenden Gebieten: [Teil 2]. - Ber. Bayer. Bot. Ges. 60: 73-83.

Lövkvist, B. \& Hultgård, U. M. 1999: Chromosome numbers in south Swedish vascular plants. Opera Bot. 137: 1-42.

Marchi, P., Illuminati, O., Macioce, A., Capineri, R. \& D'Amato, G. 1983: Genome evolution and polyploidy in Leucanthemum vulgare Lam. aggr. (Compositae). Karyotype analysis and DNA microdensitometry. - Caryologia 36: 1-18.

Morton, J. K. 1977: A cytological study of the Compositae (excluding Hieracium and Taraxacum) of the British Isles. - Watsonia 11: 211-223.

- 1981: Chromosome numbers in Compositae from Canada and the U.S.A. - Bot. J. Linn. Soc. 82: 357-368.

Nagl, W. \& Ehrendorfer, F. 1974: DNA content, heterochromatin, mitotic index, and growth in perennial and annual Anthemideae (Asteraceae). - Pl. Syst. Evol. 123: 35-54.

Parfenov, V. I. \& Dmitrieva, S. A. 1987: Kariologicheskaja kharakteristika predstavitelej flory sosudistykh rastenij Berezinskogo Biosfernogo Zapovednika. II. - Zapov. Belorussii Issl. 11: 62-69.

— \& - 1988a: Pervichnyj citogeneticheskij monitoring flory Belorussii. Izv. Akad. Nauk Azerbajdzansk. SSR. - Ser. Biol. Nauk 6: 8-11.

— \& - 1988b: Kariologicheskaja kharakteristika predstavitelej flory sosudistykh rastenij Berezinskogo biosfernogo zapovednika. - Zapov. Belorussii Issl. 12: 3-8.

Parfitt, B. D. 1981: Reports. [In Löve, A. (ed.) IOPB Chromosome number reports LXXI]. - Taxon 30: $515-516$.

Pogan, E. 1982: Further studies in chromosome numbers of Polish angiosperms. Part XVI. - Acta Biol. Cracov., Ser. Bot. 24: 159-189.

—, Czapik, R., Jankun, A. \& Kuta, E. 1982: Further studies in chromosome numbers of Polish angiosperms. Part XV. - Acta Biol. Cracov., Ser. Bot. 24: 113-126.

Polatschek, A. 1966: Cytotaxonomische Beiträgezur Flora der Ostalpenländer. II. - Österreich. Bot. Zeitschr. 113(1): 101-147.

Pólya, L. 1949: Chromosome numbers of some Hungarian plants. - Acta Geobot. Hungarica 6(2): 124-137. 
Probatova, N. S. 2000. Chromosome numbers in some plant species from the Razdolnaya (Suifun) river basin (Primorsky Territory). - Bot. Zhurn. (Moscow \& Leningrad) 85(12): 102-107.

Probatova, N. S., Sokolovskaya, A. P. \& Rudyka, E.G. 1989: Chromosome numbers in some species of vascular plants from Kunashir Island (the Kuril Islands). - Bot. Zhurn. (Moscow \& Leningrad) 74: 1675-1678.

Pustahija, F., Brown, S. C., Bogunić, F., Bašić, N., Muratović, E., Ollier, S., Hidalgo, O., Bourge, M., Stevanović, V. \& Siljak-Yakovlev, S. 2013: Small genomes dominate in plants growing on serpentine soils in West Balkans, an exhaustive study of 8 habitats covering 308 taxa. - Plant Soil 373: 427-453.

Rosselló, E. J. A., Zapatero, M. A. G. \& Andrés, F. N. 1987: Estudios cariológicos en vegetales nemorales del CW Español, II. Stud. Bot. Univ. Salamanca 6: 159-162.

Rostovtseva, T. S. 1979: Chromosome numbers of some species of the family Asteraceae Dumort. Bot. Zhurn. SSSR 64(4): 582-589.

Santos, A.C. dos. 1945: Algumas contagens de cromosomas nos gêneros Genista L. e Cytisus L. Bol. Soc. Broteriana, Ser. 2A, 19: 519-521.

Semerenko, L. V. \& Blazhevich, P. Y. 1979: Analysis of karyotypes of some species (Fabaceae Lindl.) of the Byelorussian flora. - Izv. Akad. Nauk. Belorussk SSR. Ser. Biol. Nauk (Minsk). (In Russian).

— \& Shvets, I. V. 1989: Karyologicheskoe isuchenie bobovykh (Fabaceae) Belorussii. Pp. 66-68 in Tesizy II Symp. Plant Karyology.

Siljak-Yakovlev, S., Pustahija, F., Šolić, E.M., Bogunić, F., Muratović, E., Bašić, N., Catrice, O. \& Brown, S. C. 2010: Towards a genome size and chromosome number database of Balkan flora: C-values in 343 taxa with novel values for 242. - Adv. Sci. Lett (U.S.A.) 3(2): 190-213.

Skalińska, M., Banach-Pogan, E. \& Wcisło, H. \& al. 1957: Further studies in chromosome numbers of Polish angiosperms. - Acta Soc. Bot. Polon. 26(1): 215-245.

Sopova, M. \& Sekovski, Z. 1982: Chromosome atlas of some Macedonian angiosperms. - III. God. Zborn. Biol. Fak. Univ. Kiril Metod. 35: 145-161.

Sosnovec, A. A. 1960: K citologii roda Scorzonera L. - Bot. žurn. SSSR 45: 1813-1815.

Stepanov, N. V. 1992: Chromosome numbers in representatives of some families of higher plants. Bot. Zhurn. (Moscow \& Leningrad) 77(2): 113-114.

— \& Muratova, E. N. 1995: Chromosome numbers of some taxa of higher plants of Krasnoyarsk territory. - Bot. Zhurn. (Moscow \& Leningrad) 80(6): 114-116.

Strid, A. \& Andersson, I. A. 1985: Chromosome numbers of Greek mountain plants. An annotated list of 115 species. - Bot. Jahrb. Syst. 107: 203-228.

— \& Franzen, R. 1981: Reports. [In Löve, A. (ed.) IOPB Chromosome number reports LXXIII]. Taxon 30: 829-842.

— \& - 1983: Chromosome numbers in flowering plants from Greece. - Willdenowia 13: 329-333.

Tarnavschi, I. T. 1938: Karyologische Untersuchungen an Halophyten aus Rumänien im Lichte zytoökologischer und zyto-geographischer Forschung. - Bul. Fac. Stiint. Cernauti 12: 68-106.

Tasenkevitch, L. A., Vysotskaja, E. I. \& Vorobetz, N. K. 1989: Chromosome numbers in rare and endemic species of vascular plants from the Ukrainian Carpathians. - Bot. Zhurn. (Moscow \& Leningrad) 74: 1669-1670.

Teppner, H. 1980: Karyologie und systematik einiger Gefabpilanzen der Ostalpen. - Phyton (Horn) 20: 73-94.

Tischler, G. 1934: Die Bedeutungen der Polyploidie für die Verbreitung der Angiospermen, erläutertvan den Arten Schleswig-Holsteins, mit Ausblicken auf andere Florengebiete. - Bot. Jahrb. 67: 1-36.

Van Loon, J. C. \& Setten, A. K. V. 1982: Reports. [In Löve, A. (ed.) IOPB Chromosome number reports LXXVI]. - Taxon 31: 589-592. 
Verlaque, R., Seidenbinder, M. \& Reynaud, C. 1987: Recherches cytotaxonomiques sur la spéciation en région Méditerranéenne III: espèces aneuploides. - Biol.-Ecol. Medit. 10: 315-346.

Vermeulen, P. 1949: Varieties and forms of Dutch orchids. - nederl. Krudk. Arch. 56: 204-242.

Vesely, P., Bures, P., Smarda, P. \& Pavlicek, T. 2011: Genome size and DNA base composition of geophytes: the mirror of phenology and ecology? - Ann. Bot. 109: 65-75.

Vogt, R. 1991: Die Gattung Leucanthemum Mill. (Compositae) auf der Iberischen Halbinsel. Ruizia 10: 1-261.

— 2000. In Dobea, C. \& Vitek, E. (eds), Documented Chromosome Number Checklist of Austrian Vascular Plants. - Verlag des Naturhistorischen Museums Wien, Vienna.

Zonneveld, B. J. M., Leitch, I. J. \& Bennett, M. D. 2005: First nuclear DNA amounts in more than 300 angiosperms. - Ann. Bot. 96(2): 229-244.

Žukova, P. G. 1964: Kariologičeskaja harakteristika nekotorih vidov Compositae v PoljarnoAljpijskom botaničeskom sadu. - Bot. Žurn. SSSR 49(11): 1656-1659.

Addresses of the authors:

Fatima Pustahija ${ }^{1}$, Neđad Bašić ${ }^{1} \&$ Sonja Siljak-Yakovlev ${ }^{2}$,

${ }^{1}$ Faculty of Forestry, University of Sarajevo, Zagrebačka 20, Sarajevo, Bosnia and Herzegovina. E-mails: f.pustahija@sfsa.unsa.ba; n.basic@sfsa.unsa.ba

${ }^{2}$ Ecologie, Systématique et Evolution, Univ. Paris-Sud, CNRS, AgroParisTech, Université Paris-Saclay, 91405 Orsay cedex, France. E-mail: sonia.yakovlev@u-psud.fr 